Creencias de profesores universitarios sobre la enseñanza-aprendizaje de cursos B-Learning. Revisión bibliográfica

\title{
edmetic
}

Revista de Educación Mediática y TIC

Creencias de profesores universitarios sobre la enseñanza-aprendizaje de cursos B-Learning. Revisión bibliográfica

University teachers' beliefs about teaching and learning of B-Learning courses.

A bibliographic review

Fecha de recepción: 20/06/2016

Fecha de revisión: 10/07/201X

Fecha de aceptación: 17/10/2016 


\section{Creencias de profesores universitarios sobre la enseñanza-aprendizaje de cursos B-Learning. Revisión bibliográfica}

\section{University teachers' beliefs about teaching and learning of B-Learning courses. A bibliographic review}

\section{Blanca Isela Robles Haros ${ }^{1}$, María Teresa Fernández Nistal ${ }^{2}$ \& Javier José Vales García $^{3}$}

\section{Resumen:}

El presente trabajo tiene por objetivo realizar una revisión bibliográfica sobre los estudios de las creencias de los profesores acerca de la enseñanza y aprendizaje en la modalidad Blended Learning (B-Learning). A partir de su presencia en el contexto educativo, el B-Learning emerge como una modalidad con identidad propia. Con base a su evolución, circula por contextos configurados de manera presencial y virtual, combinando métodos y estrategias de ambas modalidades. Esta modalidad ha adquirido diversas representaciones en su desarrollo, manifestando un acervo de conocimiento que debe ser compartido y sistematizado para referencia de otros contextos. Sin embargo, existe poca información en cuanto a las creencias que poseen los profesores sobre el proceso de enseñanza-aprendizaje que se sigue en esta modalidad. Entre las principales conclusiones que arrojó esta revisión, se encuentran que existe muy pocos estudios realizados a nivel mundial y menos aún localizados en Latinoamérica, lo cual dificulta el emprendimiento de las innovaciones educativas, utilizando las modalidades emergentes.

Palabras claves: Blended learning, creencias, educación superior, proceso enseñanza-aprendizaje.
Abstract:
The present study aims to conduct a bibliographic review of studies of teachers' beliefs about teaching and learning in Blended Learning (B-Learning) mode. From its presence in the educational context, the Blended Learning emerges as a modality with its own identity. Based on its evolution, it circulates through contexts configured so face-to-face and virtual, combining methods and strategies of both modalities. This modality has acquired different representations in its development, manifesting a body of knowledge that must be shared and systematized for reference in other contexts. However, there is scarce information regarding the beliefs held by teachers on the teaching- learning process that is followed through this mode. Among the main conclusions of this review is that there are very few studies conducted worldwide and located in Latin America alone, which makes difficult the development of educational innovations using emerging modalities.

\footnotetext{
1 Instituto Tecnológico de Sonora, (México); blanca.robles.19@gmail.com

2 Instituto Tecnológico de Sonora, (México); maria.fernandez@itson.edu.mx

3 Instituto Tecnológico de Sonora, (México); javier.vales@itson.edu.mx
} 
Creencias de profesores universitarios sobre la enseñanza-aprendizaje de cursos B-Learning. Revisión bibliográfica

Keywords: Blended learning, beliefs, higher education, teaching-learning process. 


\section{Introducción}

En este artículo se presenta una revisión bibliográfica sobre las creencias que poseen los profesores universitarios del proceso de enseñanza-aprendizaje en la modalidad B-Learning, para ello se realiza una conceptualización de los temas relacionados como son las creencias del profesorado y la modalidad en sí.

En la línea de investigación del pensamiento docente se han utilizado diversos términos para referirse a las concepciones de los maestros relacionadas con la actividad en su proceso educativo: teorías implícitas (Pozo, Schever, Mateos y Pérez, 1993), filosofías pedagógicas (Simmons, et al., 1999), conocimiento práctico (Van Driel, Beijaard y Verloop, 2001) y creencias (Pajares, 1992; Richardson, 1996) que es el concepto que se utilizará con mayor frecuencia a lo largo del presente trabajo. Estas se definen como construcciones psicológicas formadas por ideas, comprensiones, imágenes o proposiciones que se consideran verdaderas, dirigen las acciones de las personas y actúan como guías para la interpretación de nuevas situaciones (Kagan, 1992; Pajares, 1992; Van Driel et al., 2007). Son estructuras mentales que se obtienen desde una edad temprana y duran toda la vida; se van formando mediante la experiencia (Andrade, 2013).

Como objeto de estudio, las creencias han sido abordadas desde diversas disciplinas como la Antropología, la Sociología, la Psicología Social y la Filosofía entre otras. Debido a esto es posible encontrar una mezcla de perspectivas que dan cuenta de sus características principales (Andrade, 2013). Sin embargo, en este artículo se hace referencia a las creencias en el ámbito educativo y más concretamente a las creencias docentes en ambientes virtuales, ya que son las que nos interesa examinar.

Los estudios que consideran a las creencias en el ámbito educativo, en su mayoría se enfocan a cuestiones como la enseñanza y los procesos cognitivos, la planificación de la enseñanza, evaluación, valoración y toma de decisiones, cuestiones pedagógicas (Valle y Nuñez, 1989; Elizalde, 2002; Moreno y Azcárate, 2003; Herrera, 2010; Inguanzo-Arteaga, 2010; Andrade, 2013; Estévez-Nenninger, Valdés-Cuervo, Arreola-Olivarría y Zavala-Escalante, 2014). Sin embargo, poco se ha dicho acerca del uso de las TIC dentro el aula 
y menos dentro del contexto mexicano, solo se han identificado una cantidad muy limitada (Gómez, Farfan y Montiel, 2009; Ursani, Sánchez y Oredain 2004).

Por otra parte el B-Learning es una de las modalidades emergentes que requiere mayor investigación desde diferentes perspectivas como es la psicológica. Consiste en una mezcla o combinación de métodos de enseñanza y aprendizaje, que incluye actividades presenciales junto con el uso de herramientas en línea (Bartolomé, 2004; Caravias, 2014; Graham, Allen y Ure, 2005; Ramírez-Martinell y Maldonado, 2015).

Existen diferentes términos para denominar la modalidad B-Learning, la mayoría de los autores lo han denominado como: blended learning o abreviado con el prefijo B-Learning, aprendizaje semipresencial, aprendizaje mixto/híbrido, virtual-presencial (Cabero, 2010). Morán (2012) incluye dos denominaciones: flexible y mezclada.

Algunos autores atribuyen el surgimiento del B-Learning al fracaso en las expectativas que el E-Learning había creado en sus inicios en la Educación Superior (ES), (Bartolomé, 2004; Morán, 2012; Turpo, 2010). Se pretendía por un lado reducir los costos, mejorar la calidad de los resultados del aprendizaje y subsanar la ausencia del contacto humano, el cual influye en la motivación para permanecer en los cursos únicamente en línea (Bartolomé, 2004).

Existen controversias sobre cuándo y dónde se empezó a utilizar el BLearning (Turpo, 2010). Algunos autores mencionan que nació a inicios del siglo XXI, a partir de una sobreoferta de cursos de posgrado a distancia de las llamadas empresas DotCom (Punto Com) surgidas de manera indiscriminada en todo el mundo, pero especialmente en la región de América Central, donde se utilizaron más las teorías, tendencias y modalidades educativas (Aiello y Cilia, 2004).

Durante la última década se han realizado investigaciones sobre esta modalidad educativa, con el objetivo de identificar su impacto en la ES, los resultados de estas investigaciones son contradictorios. Algunos estudios han encontrado que esta modalidad es exitosa (Soler, Antúnez, Ramírez y Rodríguez, 2012), específicamente en cuanto a su eficiencia y economía. Por otra parte, otros estudios han encontrado que la aplicación de esta modalidad en la ES presenta diversos problemas, como la falta de 
capacitación docente (Abi Samra, Pérez y Castillo, 2010; Álvarez, 2010), de entrenamiento de los estudiantes para que se pueda presentar en ellos el aprendizaje significativo (Abi Samra, Pérez y Castillo, 2010) y un enfoque no situado en las necesidades de aprendizaje de los estudiantes, sino en el cumplimiento de los programas educativos (Fernández, 2011).

A pesar de la emergencia de esta modalidad educativa, existen investigaciones sobre lo que piensan los docentes acerca del proceso de enseñanza-aprendizaje implicado en esta modalidad. A continuación, se presenta una revisión del proceso que ha tenido el paradigma del pensamiento del profesor, sobre las creencias en el proceso de enseñanzaaprendizaje, con base en los enfoques descritos por Pérez, Mateos, Schever y Martín (2006), posteriormente una revisión bibliográfica sobre este tema en la modalidad B-Learning.

\subsection{El paradigma del pensamiento del profesor y las creencias de los profesores sobre el proceso de enseñanza-aprendizaje}

En el paradigma del pensamiento del profesor se concibe al docente como un sujeto reflexivo y racional que toma decisiones, emite juicios, posee creencias y genera prácticas para su desarrollo profesional (Clark y Peterson, 1990). Se entiende, entonces, que el pensamiento del profesor guía y orienta su conducta.

El pensamiento y las creencias que los docentes sostienen acerca de la enseñanza y el aprendizaje tienen una repercusión en sus prácticas y, por tanto, en el aprendizaje de los estudiantes. El pensamiento del docente afecta o beneficia su planificación y toma de decisiones y forman el contexto psicológico de la enseñanza (Clark y Peterson, 1990), ya que en la mente de los docentes se crea un modelo de qué, cómo y cuándo se enseña.

La perspectiva que tiene el docente sobre su rol combina creencias, intenciones, conceptos, interpretaciones y conductas que actúan continuamente y se modifican a través de la interacción social. No se espera que los profesores cambien su modo de proceder en la enseñanza, sino que re-piensen o re-describan sus formas de enseñar pretendiendo comprender cuándo y porqué funcionan (Pozo, Schever, Mateos y Pérez, 2006). 
Una descripción de los diferentes enfoques de este paradigma se presenta en el capítulo de Pérez et al. (2006), quienes identifican en su texto seis enfoques: Metacognición, Teoría de la Mente, Creencias Epistemológicas, Fenomenografía, Teorías Implícitas, así como el Perfil del Docente y Análisis de la Práctica.

La Metacognición, tiene por objetivo analizar el conocimiento consciente y el control de los procesos cognitivos. Pérez et al. (2006), mencionan que la principal aportación de este enfoque consiste en haber puesto de manifiesto que las personas no solo elaboramos conocimientos sobre los fenómenos del mundo físico y del mundo social en el que vivimos si no que, además, nos interesamos por los fenómenos del mundo psicológico y mental tanto propio como ajeno, esto nos lleva a elaborar conocimientos sobre como percibimos, comprendemos, aprendemos, recordamos y pensamos. Flavell da a conocer las primeras aproximaciones a este concepto, quien define a la metacognición como "el conocimiento que uno tiene acerca de los propios procesos y productos cognitivos" (Flavell, 1976, p. 232, citado en Pérez et al., 2006).

El enfoque de la Teoría de la Mente, por su parte, tiene por objetivo analizar el origen y la formación de la concepción implícita de la mente y su funcionamiento, es por ello que se centra en etapas más tempranas y considera los dominios psicológico e interpersonal. Los principales autores que contribuyeron a este enfoque fueron Premack y Woodruff (1978) y Dennet (1978) quienes definieron la teoría de la mente como la unión de representaciones, en cuanto a los estados, contenidos y procesos mentales que experimentan las personas individualmente y que forman parte de su conducta e interacción social. Spelke (1991) hace una aportación muy valiosa a este enfoque, ya que menciona que los bebés en su primer año de vida no actúan mecánicamente, sino de manera intencional. Años más tarde Strauss, Ziv y Stein (2002) propusieron la inclusión de los conocimientos procedimentales, aparte de los declarativos.

En cuanto al enfoque de las Creencias Epistemológicas, cuyo objetivo es analizar las creencias sobre qué es el conocimiento y el conocer, se sabe que aunque no se considera a las creencias epistemológicas como 
concepciones sobre el aprendizaje y la enseñanza, la naturaleza de su conocimiento influye en los medios que se emplean para aprender (Pérez et al., 2006). Las creencias epistemológicas se definen como ideas sobre la naturaleza del conocimiento y la manera de conocer -más o menos implícitaspor distintas personas (Hofer y Pintrich, 1997; Pintrich, 2002, citados en Pérez et al., 2006)

La Fenomenografía tiene por objetivo analizar la manera personal en que se viven o interpretan explícitamente las experiencias de enseñanzaaprendizaje. Más que en las concepciones individuales, el enfoque fenomenográfico se centra en las formas en que el aprendizaje es experimentado e interpretado, la descripción en este enfoque queda a niveles superficiales y evidentes, se constituyen a través de las interpretaciones que las personas elaboran en respuesta a demandas específicas (Pérez et al., 2006; González-Ugalde, 2014). En algunos estudios se hace referencia a la fenomenología, sin embargo para uso del presente y debido a que las investigaciones analizadas retoman este concepto de manera más reciente, se maneja como fenomenografía.

Por otra parte, el objetivo del enfoque de las Teorías Implícitas es analizar las concepciones implícitas sobre la enseñanza-aprendizaje como estructuras representacionales conscientes y coherentes. Las teorías implícitas se definen como "un conjunto de principios que restringen tanto nuestra forma de afrontar como de interpretar 0 atender las distintas situaciones de enseñanza-aprendizaje a las que nos enfrentamos" (Pérez et al. 2006, p. 79).

Por último, el enfoque del Perfil del Docente y Análisis de la Práctica, tiene como objetivo analizar la planificación y acción de enseñar, del pensamiento del profesor y de sus reflexiones sobre la propia práctica. Además es necesario mencionar que cada vez se ha tomado mayor conciencia que las concepciones y la práctica son dos aspectos que no se pueden separar dentro del proceso de enseñanza (Pérez et al., 2006).

Para tener una idea más clara de la relación que existe entre las concepciones y la práctica, Pérez et al. (2006) presentan tres aportaciones sobre esta línea: (a) el paradigma proceso-producto el cual analiza la relación entre conductas específicas de profesores y los rendimientos de los alumnos 
Creencias de profesores universitarios sobre la enseñanza-aprendizaje de cursos B-Learning. Revisión bibliográfica

(Broophy y Good, 1986); (b) la instrucción directa es otra aportación sobre las estrategias instruccionales que pudieran ser más adecuadas; y (c) el paradigma del pensamiento del profesor, que entiende al docente como un sujeto reflexivo y racional que toma decisiones, emite juicios, tiene creencias y genera rutinas propias de su desarrolla profesional (Clark y Peterson, 1986).

\section{Revisión de la literatura acerca de las creencias de profesores sobre la enseñanza-aprendizaje de cursos B-Learning}

La revisión de la literatura acerca de las creencias y/o concepciones del profesorado universitario sobre el proceso de enseñanza-aprendizaje en la modalidad B-Learning, permitió identificar una serie de estudios realizados en distintos países, como Australia, Canadá, China, Grecia y México (Ellis, Steed y Applebee, 2006; McConnell y Zhao, 2006; McShane, 2007; Lameras, Levy, Paraskasis y Webber, 2012; Roberts, 2003; Stacey y Weisenberg, 2007; Gómez, Farfan y Montiel, 2009; Ursani, Sánchez y Oredain 2004). Respecto a la metodología, la mayoría de estos estudios han aplicado métodos cualitativos y el estudio de caso.

Roberts (2003) realizó un estudio fenomenológico en una Universidad Escocesa de Reino Unido, con el fin de identificar en profesores universitarios las concepciones y aproximaciones de enseñanza usando la Web. A través de un enfoque mixto aplicó un cuestionario en línea a 256 profesores y realizó entrevistas a 17 de ellos. Los resultados que arrojaron las encuestas fueron:

- La mayoría de los participantes mencionaron que usan la Web para mantenerse informado y al día.

- Un bajo porcentaje de participantes mencionaron que utilizan la Web para la construcción de sus materiales didácticos.

- En cuanto al uso del e-mail en el diálogo entre el profesor y el estudiante, un alto porcentaje de participantes mencionaron que lo usan para cuestiones administrativas y no tanto para actividades de enseñanzaaprendizaje.

- La mayoría de los participantes reportaron una experiencia limitada en el uso de la Web en el proceso de enseñanza-aprendizaje.

- La mayoría de los participantes usan la Web para acceder a recursos 
de internet y consultar el e-mail.

Los resultados de las entrevistas muestran 3 orientaciones de enseñanza: (a) una centrada en el profesor y en los contenidos escolares; (b) centrada en el estudiante y su aprendizaje; y (c) basada en la interacción estudiante-tutor. En las dos primeras concepciones se distinguen diversas concepciones de enseñanza. En la orientación centrada en el profesor identificaron la concepción impartir información y transmitir información estructurada. En la orientación centrada en el estudiante identificaron las concepciones: facilitación de aprendizaje, desarrollo intelectual y cambio conceptual.

McShane (2004), a partir de un estudio de caso analizó el cambio del auto concepto del rol como académico que presentan profesores universitarios que combinan las modalidades de enseñanza en línea y cara a cara durante un semestre, mediante las experiencias personales de cinco profesores australianos que basaron su enseñanza mediante la utilización de un sistema de gestión de aprendizaje en línea (Web CT o Top Class), para organizar los contenidos de aprendizaje en línea de sus estudiantes. Los temas emergentes que surgieron del análisis de las entrevistas fueron:

- Relaciones mejoradas con los estudiantes

- La planificación y la enseñanza se convierten en tareas muy conscientes

- La expansión, extensión, aumento (tiempo y espacio)

- Escrutinio y reflexividad

- La centralidad de la docencia

En cuanto a los resultados de esta investigación, los académicos fueron más conscientes de su enseñanza como resultado de las decisiones que tuvieron que hacer en la combinación de modalidades presenciales y en línea. Los profesores que integraron la comunicación mediada por ordenador en su enseñanza, se mostraron notablemente con más autocontrol de sus acciones en el marco del escrutinio de sus estudiantes y otros usuarios.

Los cinco académicos en este grupo estuvieron entusiasmados con la enseñanza basada en la Web, incluso mencionaron que actualmente presentan ese entusiasmo al momento de llevar a cabo el proceso de enseñanza-aprendizaje en su campus.

edmetic, 5(2), 2016, E-ISSN: 2254-0059; pp. 94-XX

(c) edmetic, Revista de Educación Mediática y TIC 
McConnell y Zhao (2006), mediante un estudio cualitativo, analizaron las conceptualizaciones de 24 maestros chinos sobre el proceso de enseñanzaaprendizaje en línea (E-Learning y E-Teaching), las creencias que sostienen sobre su práctica en línea, las estrategias de implementación del E-Learning, los problemas a los que se enfrentan en la incorporación de E-Learning en sus cursos y sus precepciones acerca de los aprendices en línea.

A partir de un análisis de las entrevistas basado en la teoría fundamentada, se identificaron las siguientes categorías preliminares sobre las concepciones de los maestros en la modalidad E-Learning:

- La centralidad de la conferencia: Los docentes dijeron que prefieren la conferencia y consideran que es el método mediante el cual existe un mayor dominio de material teórico y produce resultados de aprendizaje de buena calidad.

- El aprendizaje cooperativo en línea: El análisis de las entrevistas mostró que los docentes universitarios chinos piensan que la introducción del aprendizaje cooperativo en línea en su práctica docente ayuda al proceso de enseñanza- aprendizaje.

- Red de aprendizaje: Los entrevistados hablaron de aprendizaje de red como una forma de ofrecer cursos para los estudiantes que están fuera del campus. En cuanto a la capacidad para "mejorar" el aprendizaje, mencionaron que se considera rápida y conveniente, pero no de una alta calidad.

- Aprendizaje del estudiante: En esta categoría los profesores sostuvieron que al parecer los estudiantes chinos no están bien preparados para este tipo de aprendizaje $y$, en muchos casos, todavía esperan que el profesor les enseña todo.

- Infraestructura y acceso: La situación en China con respecto a estas cuestiones es variable. Parece ser muy irregular y depender del contexto social y político de cada institución. Los profesores de educación superior que entrevistamos trabajo razonablemente bien universidades con recursos. La infraestructura y el acceso pueden tener poco o escaso apoyo, por lo que hace problemática la eficacia del E-Learning.

Los resultados de esta investigación sugieren que los profesores 
entrevistados se inclinan por una enseñanza tradicional o lo que ellos llaman instrucción cara a cara utilizando conferencias, ya que fue el método preferido de la enseñanza.

En su estudio cualitativo, Ellis et al. (2006) analizaron las concepciones de enseñanza y aprendizaje mediado (blended learning y teaching) de 22 profesores universitarios y las asociaciones entre estas concepciones y sus enfoques en el diseño de experiencias B-Learning de dos universidades Australianas. A partir de un análisis de las entrevistas basado en el enfoque fenomenográfico, se identificaron categorías descriptivas sobre las concepciones de los maestros en esta modalidad. Se realizaron tres preguntas detonantes a los profesores: ¿Qué entiende usted por blended learning?, ¿Qué quieres decir, mediante la enseñanza mixta? y ¿Cómo se aborda el diseño de experiencias combinadas de aprendizaje? Las categorías y subcategorías obtenidas en cada una de estas preguntas:

- El aprendizaje mixto (una perspectiva de los profesores en el aprendizaje del estudiante):

- Investigaciones críticas sobre los cambios en el mundo que nos rodea.

- Como la construcción activa de conocimiento.

- Como replicar formas de aprendizaje utilizando diferentes medios de comunicación.

- Como el uso de los diferentes medios de comunicación.

- Enseñanza mixta (una perspectiva de los profesores en la enseñanza):

- Como apoyo a los estudiantes a desarrollar y aplicar nuevos conceptos.

- Como el desarrollo de la comprensión del estudiante a través de la alineación de los medios de comunicación a los resultados del aprendizaje previstos.

- Como medio para proporcionar información a los estudiantes.

- Como la sustitución a las responsabilidades del maestro.

- Enfoques para el diseño de experiencias de aprendizaje combinado:

- El diseño remodela enfoques de la enseñanza.

- El diseño influye en los enfoques de la enseñanza.

- El diseño satura relaciones con la enseñanza.

- El diseño no está relacionado con la enseñanza. 
Stacey y Wiesenberg (2007) realizaron un estudio con metodología mixta en donde identificaron y compararon las percepciones sobre la modalidad en línea y presencial de 22 profesores universitarios de Canadá y Australia. La investigación se realizó mediante el uso del estudio de caso instrumental, en donde participaron diez profesores australianos y 12 profesores universitarios canadienses. Para la obtención de datos cualitativos utilizaron un cuestionario en línea de preguntas abiertas sobre la enseñanza de las filosofías y enfoques y para los datos cuantitativos aplicaron el Teaching Perspective Inventory (TPI), desarrollado y validado por Dan Pratt y John Collins de la University of British Columbia en el 2006. Este instrumento cuantitativo mide creencias, intenciones y acciones de los profesores universitarios.

Los principales resultados de esta investigación mostraron que la distribución de las puntuaciones en el TPI en ambas muestras de profesores, fue muy semejante en las dos modalidades.

- En cuanto a las percepciones de la encuesta en línea, los grupos difirieron en sus creencias acerca de lo que era posible realizar en cada modalidad:

- El grupo de la Universidad de Deakin (Australia) presentó una mayor preferencia por la enseñanza cara a cara, en cuanto al potencial práctico y activo y una mejora en las de relaciones sociales y personales.

- El grupo de la Universidad de Calgary (Canadá) mostró un puntaje más alto en cuanto a su preferencia por la enseñanza en línea, ya que consideran la transición de la enseñanza presencial a la transmitida en línea como un 'cambio de paradigma' en la enseñanza y el aprendizaje.

- Los participantes de ambas universidades coinciden en sus creencias sobre la modalidad en línea, en cuanto a que ayuda a los estudiantes a ser más auto-dirigidos y a los profesores a ser más facilitadores e innovadores.

- Los resultados de las encuestas permitieron identificar diferencias en las acciones de enseñanza aprendizaje entre la modalidad en línea y cara a cara. La mayoría de los participantes relacionan la enseñanza centrada en el maestro con la modalidad presencial y la centrada en el aprendiz con la enseñanza en línea.

- Los participantes de la Universidad de Calgary (Canadá) parecían ser 
Blanca Isela Robles Haros, María Teresa Fernández Nistal y Javier José Vales García

significativamente más "cuidadosos" (orientado hacia el refuerzo de la autoestima) en sus acciones en línea, que los participantes de la Universidad de Deakin (Australia), así como significativamente más orientados a la "reforma social" (orientado al cambio en el statu-quo) en sus acciones, tanto en modalidad presencial, como en línea en las aulas.

- El uso del B-Learning en la enseñanza y aprendizaje fue evidente en ambos grupos. La mayoría de los participantes utilizaron en un mismo momento la modalidad presencial, así como varios recursos tecnológicos.

- El cambio en el aprendizaje en línea dio lugar a que la mayoría de los participantes se mostraran más reflexivos e innovadores, acerca de sus prácticas de enseñanza en ambas modalidades.

Lameras et al. (2012) mediante un estudio cualitativo, identificaron las concepciones y enfoques de enseñanza-aprendizaje en Entornos Virtuales de Aprendizaje (EVA) de profesores de Ciencias de la Computación en 25 profesores universitarios griegos.

A partir de un análisis de las entrevistas basado en el enfoque fenomenográfico, se identificaron las siguientes categorías preliminares sobre las concepciones de los maestros en la modalidad E-Learning: (a) la transferencia de información; (b) la aplicación y aclaración de conceptos; (c) el intercambio, desarrollo de las ideas, la exploración y distribución de los recursos; y (d) de creación de conocimiento colaborativo, el desarrollo de la conciencia y habilidades de proceso.

Este estudio sugiere que las creencias y circunstancias pedagógicas que sustentan la enseñanza cara a cara (presencial) son más influyentes en la formación con enfoques mixtos de (Virtual Learning Environments: VLE), que únicamente por los recursos y características del sistema.

Hablando del contexto iberoamericano se encontraron pocas investigaciones que abordan el tema en ambientes virtuales y de las que no se obtuvieron datos sobresalientes para la presente investigación, ya que se le dio preferencia a aquellos que arrojaran datos relevantes como los anteriormente mencionados, llevados a cabo en contextos internacionales. Además que no retoman específicamente la modalidad B-Learning (Gómez, Farfán y Montiel, 2009; Ursani, Sánchez y Oredain 2004; Caicedo-Tamayo y Rojas-Ospina, 2014). 
Validación y confiabilidad de una escala de actitudes hacia las matemáticas o en cuanto a las actitudes en disciplinas específicas utilizando computadora ().

Con el propósito de contar con un instrumento que permita obtener información acerca de las actitudes de los estudiantes hacia las matemáticas y las matemáticas enseñadas con computadora, se elaboró la escala AMMEC (Actitudes hacia las Matemáticas y las Matemáticas Enseñadas con Computadora). En este artículo se presentan los pasos seguidos para su validación y se proporcionan datos que demuestran su confiabilidad y validez. En estos momentos, en los que el uso de la tecnología para apoyar la enseñanza de las matemáticas es cada vez más fuerte, esta escala permite monitorear cómo van cambiando las actitudes de los estudiantes hacia esta disciplina. Se incluyen resultados preliminares relativos a las actitudes de los estudiantes.

\section{Método}

Para la composición de la presente revisión bibliográfica, se realizó la exploración de estudios que consideran el tema como línea directa de investigación, como creencias, concepciones o incluso actitudes y su relación con la modalidad B-Learning, estos enfocados al contexto educativo como requisito indispensable. Se analizan documentos, que en su mayoría contienen trabajo empírico, por medio de investigación de campo y en un gran porcentaje aplicado en profesores como sujetos de investigación y en su mayoría dirigidos a nivel superior.

El procedimiento de identificación de los estudios consistió en introducir las palabras claves en español: Blended learning, creencias, proceso enseñanza-aprendizaje; y en inglés: Blended learning, beliefs, teachinglearning process en bases de datos como: Redalyc, scielo, ERIC y en la literatura científica nacional e internacional que aparece en google académico (google scholar) de manera general, esto con la finalidad de identificar los artículos que incluyan, tanto a las creencias, como al B-Learning, para conformar el estado del arte de este tema.

En la revisión en el contexto iberoamericano se detectaron artículos que 
hablaban sobre las creencias en disciplinas específicas, su importancia, efecto positivo o negativo en los estudiantes, etc. (20 estudios aproximadamente). Por otro lado varios estudios retoman la modalidad B-Learning, su importancia de implementación, casos de éxito o de fracaso (alrededor de 40). Sin embargo las investigaciones en las cuales se centra esta revisión son los que retoman ambos aspectos, de los cuales solamente se describen ocho que son los que cumplen con el objetivo por el cual se realiza la presente investigación.

Los tipos de documentos sobre los que se establece el presente estado del arte de creencias y/o concepciones sobre el proceso de enseñanzaaprendizaje en modalidad B-Learning, consta de ocho artículos publicados en revistas de investigación en su mayoría internacional, debido a que en Iberoamérica aún no se han analizado a profundidad estos temas.

\section{Resultados}

Desde un punto de vista metodológico existen coincidencias en cuanto al uso de la metodología cualitativa, utilizando el estudio de caso y técnicas como la entrevista semiestructurada en la mayoría de las investigaciones analizadas. Solo dos de los estudios utilizaron metodologías mixtas: Roberts (2004) en su investigación utilizó cuestionarios y entrevistas mediante un estudio fenomenológico; Stacey y Wiesenberg (2007) aplicó cuestionario cuantitativo y un cuestionario cualitativo, ambos en línea.

La mitad de los artículos analizados, basan los estudios en un enfoque fenomenográfico (McConnell y Zhao, 2006; Lameras, Levy, Paraskasis y Webber, 2012 y Roberts, 2003). Motivo por el cual podrían ser interesantes estudios que aportaran elementos de otros enfoques, que enriquecerían esta línea de investigación.

En el contexto iberoamericano aún no se aborda de manera específica el tema en la modalidad B-Learning, sin embargo se ha desarrollado en modalidad virtual (Gómez, Farfan y Montiel, 2009) o en cuanto a las actitudes en disciplinas específicas utilizando computadora (Ursani, Sánchez y Oredain 2004).

\section{Discusiones y conclusiones}

edmetic, 5(2), 2016, E-ISSN: 2254-0059; pp. 94-XX

(c) edmetic, Revista de Educación Mediática y TIC 
El análisis de la revisión bibliografía realizada para este artículo permite concluir que existe un vacío en el conocimiento en Latinoamérica y concretamente en México sobre las creencias en el proceso enseñanza-aprendizaje en modalidad B-Learning, es por ello que se considera necesario recomendar a los investigadores la posibilidad de basar sus investigaciones en este tipo de temas emergentes.

En términos generales, los resultados de estos estudios indican que la mayoría de las concepciones de enseñanza-aprendizaje que sostienen los maestros se sitúan en una etapa de transición entre la práctica de enseñanza tradicional y la constructivista (Ellis, Steed y Applebee, 2006; McConnell y Zhao, 2006; Lameras, Levy, Paraskasis y Webber, 2012; Roberts, 2003) y que existen diferencias en las concepciones según la modalidad de enseñanza; la mayoría de los profesores sostienen concepciones centradas en el maestro en la enseñanza cara a cara y centradas en el aprendiz en la modalidad en línea (Stacey y Weisenberg, 2007).

En cuanto a las ventajas del B-learning, algunos de los profesores entrevistados en los diversos estudios coinciden en las siguientes: (a) la transferencia de información; (b) la aplicación y aclaración de conceptos; (c) el intercambio, desarrollo de las ideas, la exploración y distribución de los recursos; y (d) de creación de conocimiento colaborativo.

\section{Referencias bibliográficas}

ABI SAMRA, S., PÉREZ, T., y CASTILLO, E. (2010). B-learning en el aprendizaje de inglés con propósitos académicos, a nivel universitario. Multiciencias, 10 , 172-177.

Recuperado

de http://www.redalyc.org/articulo.oa?id $=90430360031$

AIELLO, M., y CILIA, W. (2004). El blended learning como práctica transformadora. Pixel-Bit. Revista de Medios y Educación, 23, 21-26. Recuperado de http://www.Imi.ub.es/personal/bartolome/articuloshtml/04_blended lear ning/documentacion/2_aiello.pdf

ÁLVAREZ, I. (febrero, 2010). Acercamiento al estudio de los estudiantes "exitosos" en los cursos en línea de Red Escolar. Revista e-formadores. 
http://red.ilce.edu.mx/sitios/revista/e formadores_pri_10/articulos/nadia alvarez feb2010.pdf

ANDRADE, J. (mayo-agosto, 2014). Creencias sobre el uso de las tecnologías de la información y la comunicación de los docentes de educación primaria en México. Revista Electrónica "Actualidades Investigativas en Educación", 14(2), 1-29. Recuperado de http://www.redalyc.org/articulo.oa?id=44731371017

ANDRADE, J. (2013). Creencias sobre el uso de las TIC de los docentes de educación primaria en México. Revista Electrónica de educación "Sinéctica", $41 . \quad$ Recuperado de https://sinectica.iteso.mx/index.php/SINECTICA/article/view/39/32

BARTOLOMÉ, A. (2004). Blended Learning. Conceptos básicos. Pixel-Bit. Revista de Medios y Educación, 23, 7-20. Recuperado de http://www.sav.us.es/pixelbit/articulos/n23/n23art/art2301.htm

BROOPHY, J., Y GOOD, T. (1986). Teacher behaviour and student achievement. En M. Wittrock (Ed.), Handbook of Research on Teaching. Nueva York: MacMillan.

CABERO, J. (dir.). (2010). Usos del E-Learning en las Universidades Andaluzas: Estado de la situación y análisis de buenas prácticas. Sevilla: Grupo de Investigación Didáctica de la Universidad de Sevilla.

CAICEDO-TAMAYO, A. M. y ROJAS-OSPINA, T. (2014). Creencias, conocimientos y usos de las TIC de los profesores universitarios. Educ. Educ., 17(3), 517533. doi: 10.5294/edu.2014.17.3.7.

CANCHÉ, J., FARFÁN, R., y MONTIEL, G. (2009). Creencias y concepciones de los profesores: Un estudio en un escenario Virtual. Comité Latinoamericano de Matemática Educativa. Recuperado de http://www.matedu.cicata.ipn.mx/archivos/(JCanche-RFarfanGMontiel2009)-ALME22-.pdf

CARAVIAS, V. (2014). Teachers' Conceptions and Approaches to Blended Learning: A Literature Review. SDIWC- Digital Library, 61-75. Recuperado de http://sdiwc.net/digital-library/teachers-conceptions-andapproaches-to-blended-learning-a-literature-review 
CLARK, C. y PETERSON, P. (1986). Teachers Thought Process. En M. Wittrock (Ed.), La investigación de la enseñanza III, (pp. 443-531). Barcelona, España: Paidós.

CLARK, C., y PETERSON, P. (1990). Procesos de pensamiento de los docentes. En M. Wittrock (Ed.), La investigación de la enseñanza III, (pp. 443-531). Barcelona, España: Paidós.

DE LA CRUZ, M., POZO, J., HUARTE, M., y SCHEUER, N. (2006). Concepciones de enseñanza y prácticas discursivas en la formación de futuros profesores. En J. Pozo, N. Schever, M. Pérez, M. Mateos, E. Martín \& M. de la Cruz (Eds.), Nuevas formas de pensar la enseñanza y el aprendizaje. Barcelona: Graó.

DENNET, D. (1978). Braimstorms. Philosophical essays on Mind and Psycology. Cambridge: MIT Press.

ELIZALDE HEVIA, A. (2002). Otro sistema de creencias como base y consecuencia de una sustentabilidad posible. En Enrique Leff. (coord.), Ética, vida y sustentabilidad (pp. 51-70). México: PNUMA.

ELLIS, R., STEED, A., y APPLEBEE, A. (2006). Teacher conceptions of blended learning, blended teaching and associations with approaches to design. Australasian Journal of Educational Technology, 22(3), 312-335. Recuperado de http://www.ascilite.org.au/ajet/ajet22/ellis.html

ESTÉVEZ-NENNINGER, E. H, VALDÉS-CUERVO, A. A., ARREOLA-OLIVARRÍA, C. G., Y ZAVALA-ESCALANTE, M. G. (2014). Creencias sobre enseñanza y aprendizaje en docentes universitarios. Magis, Revista Internacional de Investigación en Educación, 6(13), 49-64. Recuperado de http://revistas.javeriana.edu.co/index.php/MAGIS/article/view/8784

FERNÁNDEZ, N. (2011). Promoción del cambio de estilos de aprendizaje y motivaciones en estudiantes de educación superior mediante actividades de trabajo colaborativo en blended learning. Revista Iberoamericana de Educación a Distancia, 14(2), 189-208. Recuperado de

http://ried.utpl.edu.ec/images/pdfs/volum142/promociondelcambio.pdf

GONZÁLEZ-UGALDE, C. (julio-diciembre, 2014). Investigación fenomenográfica. Magis. Revista Internacional de Investigación en Educación, 7(14), 141- 
$158 . \quad$ Recuperado de http://www.redalyc.org/pdf/2810/281032883011.pdf

GRAHAM, C. R., ALLEN, S., y URE, D. (2005). Benefits and Challenges of Blended Learning Environments. In M. Khosrow-Pour (Ed.), Encyclopedia of Information Science and Technology, First Edition (pp. 253-259). Hershey, PA: Information Science Reference. doi:10.4018/978-1-59 140-553-5.ch047. HERRERA, J. (2010). Teacher beliefs and practices: Their effects on student achievement in the urban school setting. Doctoral dissertation, Kansas State University (United States), United States. Recuperado de https://krex.k-

state.edu/dspace/bitstream/handle/2097/3889/JohnHerrera2010.pdf?se quence $=7$

INGUANZO-ARTEAGA， G. (2010). Creencias de los profesores de nivel de licenciatura sobre la naturaleza del conocimiento y los procesos de enseñanza y aprendizaje. Tesis doctoral, Doctorado Interinstitucional en Educación. Puebla, México: UIA Puebla.

KAGAN, D. (1992). Implications of research on teacher belief. Educational Psychologist, 27(1), 65-70. doi: 10.1207/s15326985ep2701_6.

LAMERAS, P., LEVY, P., PARASKAKIS, I., y WEBBER, S. (2012). Blended university teaching using virtual learning environments: conceptions and approaches. Instructional Science, 40(1), 141-157. Recuperado de http://link.springer.com/article/10.1007\%2Fs1 1251-011-9170-9

MCCONNELL, D., y ZHAO, J. (2006). Chinese higher education teachers' conceptions of E-Learning: Preliminary outcomes. In Proceedings of the 23rd Annual Ascilite Conference: Who is Learning? Whose technology?. The University of Sydney. Recuperado de http://www.ascilite.org.au/conferences/sydney06/proceeding/pdf_pap ers/p224.pdf

MCSHANE, K. (2007). Integrating face-to-face and online teaching: academics' role concept and teaching choices. Teaching in Higher Education, 9(1), 3-16.

Recuperado

de http://www.tandfonline.com/doi/abs/10.1080/1356251032000155795?.jou rnalCode=cthe20\#.VHQz7luG-So

edmetic, 5(2), 2016, E-ISSN: 2254-0059; pp. 94-XX

(c) edmetic, Revista de Educación Mediática y TIC 
MORÁN, L. (marzo, 2012). Blended-Learning. Desafío y Oportunidad para la Educación Actual. Revista Electrónica de Tecnología Educativa, (39). Recuperado de: http://edutec.rediris.es/Revelec2/Revelec39/pdf/Edutece $39 \% 20$ Moran.pdf

MORENO, M. y AZCÁRATE, C. (2003). Concepciones y creencias de los profesores universitarios de matemáticas acerca de la enseñanza de las ecuaciones diferenciales. Enseñanza de las Ciencias, 21 (2), 265-280.

PAJARES, M. F. (1992). Teachers' beliefs and educational research: Cleaning up a messy construct. Review of Educational Research, 62(3), 307-332. Recuperado de http://rer.sagepub.com/content/62/3/307

PÉREZ, M., MATEOS, M., SCHEUER, N. y MARTíN, E. (2006). Enfoques en el estudio de las concepciones sobre el aprendizaje y la enseñanza. En J. Pozo, N. Schever, M. Pérez, M. Mateos, E. Martín y M. De la cruz (Eds.), Nuevas formas de pensar la enseñanza y el aprendizaje. Las concepciones de profesores y alumnos. (pp. 55-94). España: GRAO.

Pozo, J., Schever, N., Mateos, M. \& Pérez, M. (2006). Las teorías implícitas sobre el aprendizaje y la enseñanza. En J. I. Pozo et al. (Eds.), Nuevas formas de pensar la enseñanza y el aprendizaje. Barcelona: Graó.

Premack, D. and Woodruff, G. (1978). Does the chimpanzee have a theory of mind?. Behavioral and brain Sciences, 1 (4). 515-526.

RAMÍREZ-MARTINELL, A., y MALDONADO, G. (2015). Multimodalidad en educación superior. En A. Ramírez y M. Casillas (Coords), Internet en educación superior. Córdoba: Brujas. Recuperado de http://www.uv.mx/personal/albramirez/files/2014/12/hablame_de_tic2.p df $\#$ page $=20$

ROBERTS, G. (2003). Teaching using the web: Conceptions and approaches from a phenomenographic perspective. Instructional Science, 31 (1-2), 127-150.

Recuperado

de http://link.springer.com/article/10.1023\%2FA\%3A1022547619474

SIMMONS, P., EMORY, A., CARTER, T., COKER, T., FINNEGAN, B., CROCKETT, D., ET AL. (1999). "Beginning teachers: Beliefs and classroom actions". Journal of Research in Science Teaching, 36, 930-954. 
SOLER, Y., ANTÚNEZ, G., RAMÍREZ, W., y RODRÍGUEZ, Y. (noviembre, 2012). Curso de Infoctenología y Redacción Científica: El B-learning para profesionales de las Ciencias Agropecuarias. Revista Electrónica de Veterinaria, 13(11), 1-7. Recuperado de http://www.redalyc.org/articulo.oa?id=63624842018

SPELKE, E. (1991). Physical knowledge in infancy: Reflection on Piaget's theory. En CAREY, S. SPELKE, E. (Eds.), The epigenesis of mind. Hillsdale, NJ.LEA.

STACEY, E., y WIESENBERG, F. (2007). A study of face-to-face and online teaching philosophies in Canada and Australia. The Journal of Distance Education/Revue de l'Éducation à Distance, 22(1), 19-40. Recuperado de http://files.eric.ed.gov/fulltext/EJ805067.pdf

STRAUSS, S., ZIV, M., y STEIN, A. (2002). Teaching as a natural cognition and its relations to preschoolers' developing theory of mind. Cognitive Development, 17, 1473-1787.

TURPO, O. (2010). Contexto y desarrollo de la modalidad educativa blended learning en el sistema universitario iberoamericano. Revista mexicana de investigación educativa, 15(45), 345-370. Recuperado de http://www.scielo.org.mx/scielo.php?script=sci_arttext\&pid=S1405$\underline{66662010000200002}$

URSANI, S., SÁNCHEZ, G., y OREDAIN, M. (2004). Validación y confiabilidad de una escala de actitudes hacia las matemáticas enseñadas con computadora. En Educación Matemática, 16(003), 59-78. Recuperado de http://redalyc.vaemex.mx/src/inicio/ArtPdfRed.jsp?iCve=40516304

VAN DRIEL, J., BEIJAARD, D., y VERLOOP, N. (2001). Professional development and reform in science education: The role of teachers' practical knowledge. Journal of Research in Science teaching, 38(2), 137-158. Recuperado de http://onlinelibrary.wiley.com/doi/10.1002/10982736(200102)38:2\%3C137::AID-TEA1001\%3E3.0.CO:2-U/abstract

VAN DRIEL, J., BULTE, A., y VERLOOP, N. (2007). The relationships between teachers' general beliefs about teaching and learning and their domain specific curricular beliefs. Learning and Instruction, 17(2), 156-171. doi:10.1016/j.learninstruc.2007.01.010

VALLE, A., y NUÑEZ, J. (1989). Las expectativas del profesor y su incidencia en el edmetic, 5(2), 2016, E-ISSN: 2254-0059; pp. 94-XX 
Creencias de profesores universitarios sobre la enseñanza-aprendizaje de cursos B-Learning. Revisión bibliográfica

contexto institucional. Revista de Educación, 290, 293-519.

\section{Cómo citar este artículo:}

Robles Haros, B.I., Fernández Nistal, M. T., y Vales García, J.J. (2016). Creencias de profesores universitarios sobre la enseñanza-aprendizaje de cursos BLearning. Revisión bibliográfica. EDMETIC, Revista de Educación Mediática y TIC, 5(2), 94-116. 